\title{
Hepatitis E virus in sheep in Italy
}

\author{
Vittorio Sarchese $^{1}$ | Federica Di Profio ${ }^{1}$ | Irene Melegari $^{1}$ | Andrea Palombieri ${ }^{1}$ | \\ Sandra Bermudez Sanchez ${ }^{1}$ | Alessio Arbuatti ${ }^{1}$ | Massimo Ciuffetelli ${ }^{2}$ | Fulvio Marsilio ${ }^{1}$ | \\ Vito Martella ${ }^{3}$ | Barbara Di Martino ${ }^{1}$
}

${ }^{1}$ Faculty of Veterinary Medicine, Università degli Studi di Teramo, Teramo, Italy

${ }^{2}$ Azienda ASL Avezzano Sulmona L'Aquila, L'Aquila, Italy

${ }^{3}$ Faculty of Veterinary Medicine, Università Aldo Moro di Bari, Valenzano, Italy

\section{Correspondence}

Barbara Di Martino, Faculty of Veterinary Medicine, Università degli Studi di Teramo, Italy.

Email: bdimartino@unite.it

\begin{abstract}
Summary
Hepatitis E virus (HEV) is the leading cause of human enterically transmitted viral hepatitis occurring around the world both as outbreaks and as sporadic cases. The accumulating literature indicates that domestic pigs and wild boars are the main reservoirs of genotype 3 and genotype 4 for human infections in industrialized countries. However, the recent identification of HEV from various animal species poses additional potential concerns for HEV zoonotic infection.

In this study, the role of sheep as potential host of hepatitis E virus (HEV) was investigated. By screening 192 sheep from seven farms located in Abruzzo Region (Southern Italy), HEV-specific antibodies were detected in the sera of 41 animals (21.3\%) whilst the RNA of HEV, genotype 3, was detected in 20 faecal $(10.4 \%)$ and three serum samples (1.6\%). Upon sequence analyses of a partial ORF2 gene region of eight HEV positive samples, the sheep sequences all grouped together within HEV genotype 3 subtype c, being most closely related to HEV strains identified in goat and wild boar from Abruzzo. This is the first study that demonstrates, serologically and molecularly, the presence of HEV in sheep population in a European country.
\end{abstract}

KEYWORDS

antibodies, genotype 3 , hepatitis E virus (HEV), RNA, sheep

\section{1 | INTRODUCTION}

Hepatitis E virus (HEV) infection is a major health problem worldwide. World Health Organization estimations report the occurrence of 20 million HEV infections every year with 3.3 million symptomatic cases (WHO, 2018).

HEV is a small non-enveloped RNA virus classified in the genus Orthohepevirus, family Hepeviridae (Purdy et al., 2017). Based on full-length genome analysis, HEV strains are currently classified into eight genotypes (Gt1-Gt8). Four major Gts (1-4), all representing a single serotype, have been implicated in human disease. Gt1 and Gt2 are endemic in developing countries and restricted to humans, where they are predominantly transmitted through the faecal-oral route, either indirectly through contaminated drinking water or food. Gt3 and Gt4 infect humans and animals and are responsible for sporadic cases of autochthonous human hepatitis $\mathrm{E}$ in industrialized countries (Kamar et al., 2012). Pigs and wild boars are recognized as the main reservoirs of infection, although several additional animal species may act as HEV hosts, including domestic ruminants (Di Martino et al., 2016; Wu, Si, Jiang, Li, \& Jin, 2015; Yan et al., 2016).

Serological studies suggest that HEV infection is common in particular geographical regions of Italy (Lucarelli et al., 2016; Spada et al., 2018), with $48.9 \%$ (153/3131) anti-HEV IgG positivity amongst blood donors in Abruzzo region (Southern Italy); HEV Gt3c has been detected in two of these donors (Lucarelli et al., 2016). Similar HEV strains have also been found in wild boar (Aprea et al., 2018; Di Profio et al., 2016) and goat stools (Di Martino et al., 2016) collected from the same geographical area, but the ecological/epidemiological cycles of HEV across 
local human and animal populations have not been investigated thoroughly. Sheep farming for meat and milk production play an important role in Abruzzo Region. In order to gather information on their possible role as host and source of HEV for humans, in this study we investigated serologically and molecularly the epidemiology of HEV in sheep.

\section{2 | MATERIAL AND METHODS}

\section{1 | Sample collection}

Between January and June 2018, serum and faecal specimens were collected from 192 clinically healthy sheep older than 6 months from seven small farms located in the province of L'Aquila (Abruzzo Region, Italy). Sampling of herds was accomplished by random selection of a list of flocks located in an area of approximately $771 \mathrm{~km}^{2}$. In the same geographical area, HEV RNA was recently found at high rate $(20.1 \%, 29 / 144)$ in wild boars by molecular screening of liver samples (Aprea et al., 2018). Flock size ranged from 12 to 60 animals. All the sampled animals were bred for both milk and meat and were grazed freely on pastures.

\subsection{Antibody-detection ELISA}

Serological investigation for HEV infection was carried out testing all serum samples with a species-independent commercial doubleantigen sandwich Enzyme-Linked Imunnosorbent Assay (ELISA) kit (Wantai Biological, Beijing, China), based on a recombinant peptide spanning amino acids 394-604 of the open reading frame (ORF) 2 of HEV Gt1 (Wang et al., 2002) and able to detect total antibodies against all genotypes of HEV.

\section{3 | Detection of HEV RNA in serum and faecal samples}

Faecal samples were homogenized in 10\% Dulbecco's modified Eagle's medium and then centrifuged at $10,000 \mathrm{~g}$ for $3 \mathrm{~min}$. The feline calicivirus strain F9 (ATCC ${ }^{\circledR}$ VR-782 ${ }^{\text {TM }}$ ) was added to each sample as RNA extraction control, and to test for the presence of inhibitors. Total RNA was extracted individually from each serum $(0.5 \mathrm{ml})$ and faecal supernatant $(0.5 \mathrm{ml})$ by using the TRIzol LS (Invitrogen, Ltd, Paisley, UK) procedure and analysed by HEV-specific real-time reverse transcription PCR (qRT-PCR), targeting a conserved 68 nucleotide (nt) region of ORF3 gene (nt 5261-5330 on GenBank accession no. M73218), as previously described (Jothikumar, Cromeans, Robertson, Meng, \& Hill, 2006). Viral RNA quantification was performed using the SuperScript III platinum OneStep Quantitative RT-PCR system (Invitrogen Ltd, Milan, Italy) in a $25-\mu$ l volume comprising $5 \mu \mathrm{l}$ of extracted RNA and $20 \mu \mathrm{l}$ of master mix. Primers (JVHEVF: 5'-GGTGGTTTCTGGGGTGAC-3' and JHEVR: AGGGGTTGGTTGGATGAA-3') and TaqMan probe (JVHEVP: $5^{\prime}$ TGATTCTCAGCCCTTCGC- $3^{\prime}$ ) were used at concentrations of 200 and $100 \mathrm{nM}$, respectively. A standard curve was generated using $10^{2}$ to $10^{7}$ copies per reaction of HEV plasmid DNA constructed by cloning the 68 bp ORF3 fragment of a wild boar strain (HEV/WB/P6-15/ ITA, accession no. KU508285) (Di Profio et al., 2016) into pCR 2.1 vector (TA Cloning Kit, Invitrogen, Ltd, Milan, Italy).

Amplification of RNA for sequencing was attempted on all the samples containing quantifiable HEV RNA, by using a nested RT-PCR (external primer pair, Fw: 5'-CCCTTATCCTGCTGAGCATTCTC-3' and Rev: 5'-AAYTATGCWCAGTACCGGGTTG-3'; internal primer pair, Fw: 5'-GTYATGYTYTGCATACATGGCT-3' and Rev: 5'AGCCGACGAAATYAATTCTGTC-3') (Wang, Zhang, Ling, Li, \& Harrison, 2000), targeting a 345 bp portion of the ORF2 gene highly conserved amongst the four major HEV Gts (Gt1-Gt4). RT and PCR were performed in one-step procedure, using the SuperScript III one-step system (Invitrogen, Ltd, Paisley, UK), following thermal conditions previously described.

Standard precautions were taken to avoid contamination of the quantitative and qualitative RT-PCR procedures. Plasmid was prepared in a separate room, and microtubes containing each aliquot of plasmid standards were not taken into the RT-PCR set-up room. In addition to spatial separation of workspaces, each run included negative controls for RNA extraction, for qRT-PCR or RT-PCR, and for nested PCR.

\begin{tabular}{|c|c|c|c|c|}
\hline \multicolumn{5}{|c|}{ Positive/Total (\%) } \\
\hline Sheep farm & $\begin{array}{l}\text { No. animals } \\
\text { tested }\end{array}$ & HEV antibodies & Real time RT-PCR & Nested RT-PCR \\
\hline AQ1 & 60 & $23 / 60(38.3)$ & $5 / 60(8.3)$ & $2 / 60(3.3)$ \\
\hline AQ2 & 12 & $2 / 12(16.6)$ & $3 / 12(25.0)$ & $2 / 12(16.6)$ \\
\hline AQ3 & 18 & 2/18 (11.1) & $1 / 18(5.5)$ & $1 / 18(5.5)$ \\
\hline AQ4 & 19 & $3 / 19$ (15.7) & $5 / 19(26.3)$ & $2 / 19(10.5)$ \\
\hline AQ5 & 35 & 4/35 (11.4) & $5 / 35(14.3)$ & $1 / 35(2.8)$ \\
\hline AQ6 & 30 & $2 / 30(6.6)$ & $0 / 30(0)$ & N.I. \\
\hline AQ7 & 18 & $4 / 18(22.2)$ & $1 / 18(5.5)$ & $0 / 18(0)$ \\
\hline Total & 192 & 40/192 (21.3) & 20/192 (10.4) & $8 / 192(4.2)$ \\
\hline
\end{tabular}

TABLE 1 Serological and molecular prevalence of HEV in seven sheep farms in province of L'Aquila (Abruzzzo, Italy)

N.I.: not investigated. 


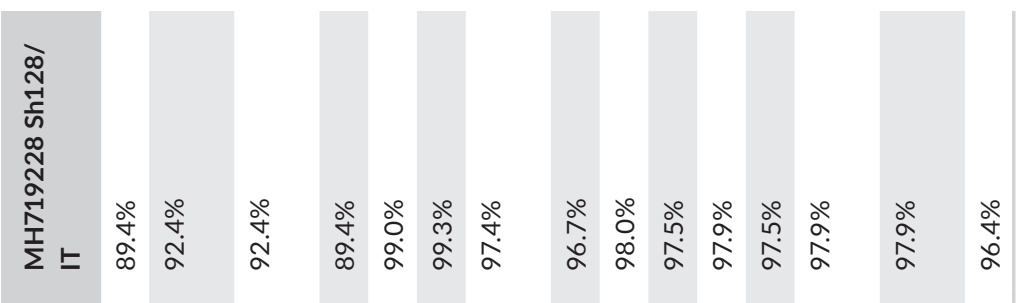

产

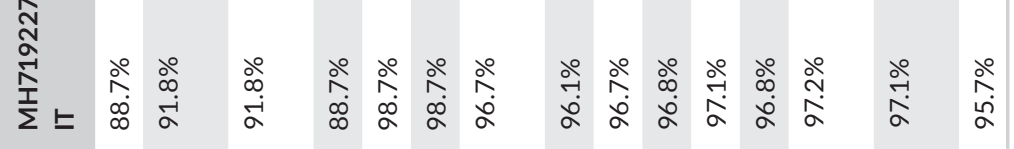

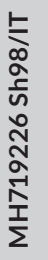

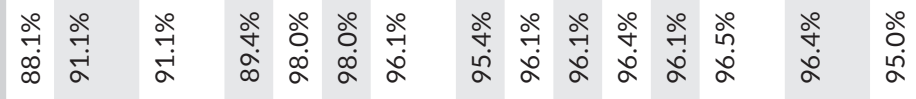
$\stackrel{2}{\stackrel{5}{\circ}}$

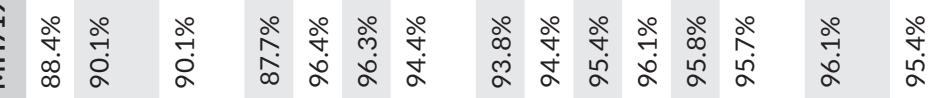

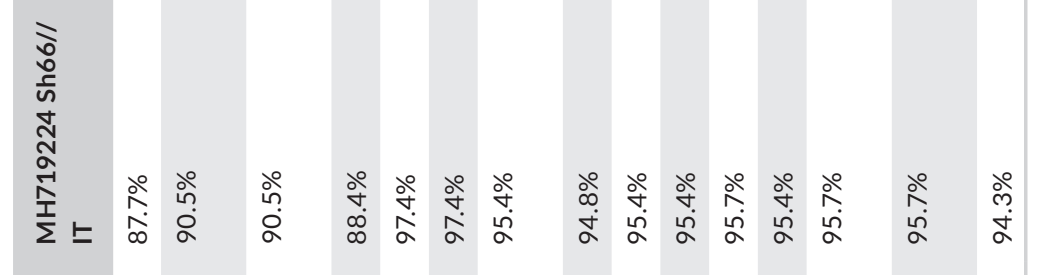

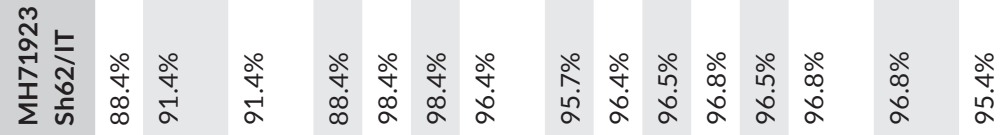

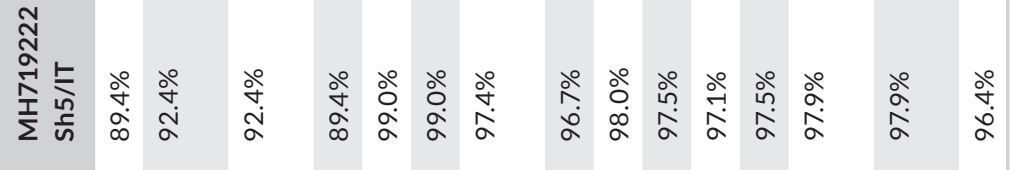

녿응

$\frac{0}{\frac{0}{0}} \frac{\pi}{\frac{\pi}{0}}$

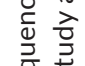

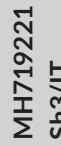

ว 


\subsection{Sequence and phylogenetic analyses}

All the amplicons were purified by using a QIAquick gel extraction kit (Qiagen $\mathrm{GmbH}$, Hilden, Germany) and subjected to direct sequencing using BigDye Terminator Cycle chemistry and 3730 DNA Analyser (Applied Biosystems, Foster, CA, USA). Basic Local
Alignment Search Tool (BLAST; http://www.ncbi.nlm.nih.gov) with default values was used to find homologous hits. Tree was generated using the Neighbour-joining method and the p-distance model with Geneious software package vers. 9 (Biomatters, New Zealand, http://www.geneious.com), supplying a statistical support with bootstrapping of 1000 replicates.

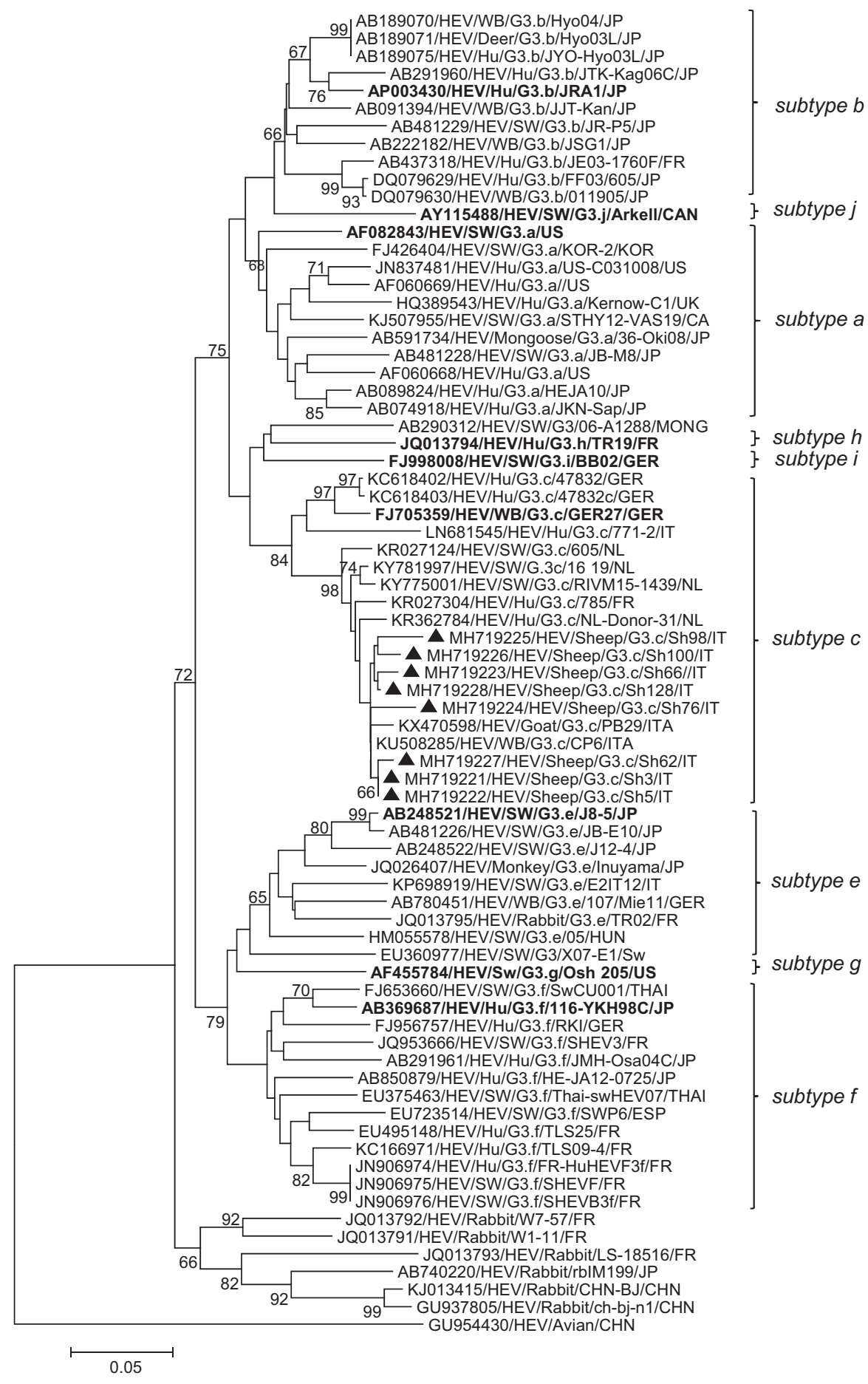

FIGURE 1 Phylogenetic tree constructed on the $0.3 \mathrm{~kb}$ at the $5^{\prime}$ end of the ORF2 gene of the sheep strains detected in this study. Tree was generated using the Neighbor-joining method and the $\mathrm{p}$-distance model supplying a statistical support with bootstrapping of 1,000 replicates. The scale bar indicates nucleotide substitutions per site. The reference strains representative of each Gt3 HEV subtype (http:// talk.ictvonline.org/ictv_wikis/hepeviridae/w/sg_hepe/343.proposed-orthohepevirus-a-subtype-reference-sequences) are in boldface. Black triangles indicate the sheep sequences detected in this study. Hu: human; SW: swine; WB: wild boar 


\section{3 | RESULTS AND DISCUSSION}

Out of 192 sheep serum samples tested, antibodies against HEV were detected in a total of 41 sera with an overall prevalence of 21.3\% (41/192; 95\% Cl 15.2-26.7\%). All seven farms were seropositive for HEV with rates ranging from $6.6 \%$ to $38.3 \%$ (Table 1 ). Molecular screening by qRT-PCR detected HEV RNA in 20/192 (10.4\%) faecal specimens and in 3/192 (1.6\%) serum samples, all deriving from animals that were also positive for the stool sample. Viral loads ranged from $2.9 \times 10^{2}$ to $5.8 \times 10^{6}$ copies/gram faeces and $1.9 \times 10^{2}$ to $2.3 \times 10^{3}$ copies $/ \mathrm{ml}$ serum (cut-off cycle threshold 38.00). HEV RNA was detected in six out of seven farms investigated with rates ranging from $5.5 \%$ to $26.3 \%$ (Table 1). The highest prevalence was detected in the farm AQ4 (26.3\%, 5/19), in which antibodies anti-HEV were detected in $15.7 \%$ (3/19) of the animals, whilst the lowest prevalence (5.5\%) was found in the farms AQ3 $(1 / 18)$ and AQ7 (1/18), in which the seropositivity rates were $11.1 \%$ (2/18) and 22.2\% (4/18), respectively. In the farm AQ6, HEV RNA was not detected. In this flock it was also observed the lowest seropositivity $(6.6 \%, 2 / 30)$. Interestingly, HEV antibodies were detected in $8 / 20$ animals that had HEV-RNA positive faecal samples and in $1 / 3$ animals with HEV-RNA positive serum samples. By using nested RT-PCR (Wang et al., 2000) viral sequences were obtained from eight faecal samples. Upon sequence analyses (GenBank accession no. MH719221-MH719228), all the ovine amplicons shared 94.4-100\% nucleotide ( $n t)$ identity to each other and displayed the highest identities (96.4-99.3\%) to the goat (PB29/ITA, KX470598) and wild boar (P6-15/ITA, KU508285) Gt3 HEV strains detected in Abruzzo (Di Martino et al., 2016; Di Profio et al., 2016). Also, high identity (93.8\%-98.0\%) was found to human Gt3 HEVs identified in blood donors and hepatitis patients from The Netherland and France (Lhomme et al., 2015; Smith et al., 2015) and to HEV strains detected in porcine blood products used in meat industry (Boxman et al., 2017) (Table 2). Nucleotide identity to Italian human Gt3 HEVs in this fragment was $77.5-89.9 \%$, with the best match (nt identities $87.7 \%-89.9 \%$ ) being to the strain Gt3 subtype c, Hu/771-2/ IT (LN681545), identified in an Italian blood donor from Abruzzo (Lucarelli et al., 2016). After phylogenetic analysis, the Italian sheep sequences segregated within the Gt3 subtype c, together with other $\mathrm{HEV}$ s identified in humans and animals, being most closely related to sequences derived from wild boar and goat stool samples detected in Abruzzo (Figure 1). The strain Hu/771-2/IT (Lucarelli et al., 2016) was more distantly related than were HEV strains from French and Dutch patients (Lhomme et al., 2015; Smith et al., 2015).

Thus far, data on the potential role of sheep as animal source of HEV infection for humans have been collected in rural areas of China located in the Shandong Province (Yan et al., 2016) and in the Xinjiang Region (Wu et al., 2015). HEVs belonging to the Gt4, that is predominant in Asia, were detected in liver and stool samples of sheep. The results obtained in this study provide firm evidence for the presence of Gt3 HEVs in sheep in Italy. These findings, along with the detection of HEV in goats (Di Martino et al., 2016), underline the importance of small domestic ruminants in the epidemiology of HEV, markedly in the Region investigated in this study. Furthermore, the circulation of highly genetically similar strains amongst sheep, goats, wild boars and humans suggests that multiple inter-species transmission of HEV might be occurring in the surveyed area.

Considering the importance of sheep as livestock animals in Southern Italy, characterized by an ideal environment for shepherds and their flocks, surveillance for HEV infections in potential risk groups, such as farmers and slaughterhouse workers, would be required. Raw or/and undercooked pork meat are the most frequently reported food products associated with outbreaks of hepatitis $\mathrm{E}$. In Southern Italy sheep is used to prepare some milk and meat products such as fresh cheese and skewered mutton. It will be necessary to assess whether consumption of undercooked sheep meat or milk products could pose a risk for human health.

\section{ACKNOWLEDGEMENTS}

This study was supported by grants from the University of Teramo, Italy, and from the Italian Ministry of University. Sandra Bermudez Sanchez is granted by Marie Skłodowska-Curie COFUNDREP-EAT 713714: Innovative Research and Training Doctoral Programme in the interdisciplinary domain of food and healthy diet.

\section{CONFLICT OF INTEREST}

All Authors declare that there are no financial or other relationships that might lead to a conflict of interest. All authors have seen and approved the manuscript and have contributed significantly to the work.

\section{ORCID}

Barbara Di Martino iD https://orcid.org/0000-0001-6192-7166

\section{REFERENCES}

Aprea, G., Amoroso, M., Di Bartolo, I., D'Alessio, N., Di Sabatino, D., Boni, A., ... Fusco, G. (2018). Molecular detection and phylogenetic analysis of hepatitis $E$ virus strains circulating in wild boars in south-central Italy. Transboundary and Emerging Diseases, 65(1), e25-e31. https:// doi.org/10.1111/tbed.12661

Boxman, I., Jansen, C., Hägele, G., Zwartkruis-Nahuis, A., Cremer, J., Vennema, H., \& Tijsma, A. (2017). Porcine blood used as ingredient in meat productions may serve as a vehicle for hepatitis $\mathrm{E}$ virus transmission. International Journal of Food Microbiology, 257, 225-231. https://doi.org/10.1016/j.ijfoodmicro.2017.06.029

Di Martino, B., Di Profio, F., Melegari, I., Sarchese, V., Robetto, S., Marsilio, F., \& Martella, V. (2016). Detection of hepatitis E virus (HEV) in goats. Virus Research, 225, 69-72. https://doi.org/10.1016/j. virusres.2016.09.008

Di Profio, F., Melegari, I., Sarchese, V., Robetto, S., Marruchella, G., Bona, M., ... Di Martino, B. (2016). Detection and genetic characterization of hepatitis $\mathrm{E}$ virus (HEV) genotype 3 subtype $\mathrm{c}$ in wild boars in Italy. Archives of Virology, 161(10), 2829-2834. https://doi.org/10.1007/ s00705-016-2964-y 
Jothikumar, N., Cromeans, T., Robertson, B., Meng, X., \& Hill, V. (2006). A broadly reactive one-step real-time RT-PCR assay for rapid and sensitive detection of hepatitis E virus. Journal of Virological Methods, 131(1), 65-71. https://doi.org/10.1016/j.jviromet.2005.07.004

Kamar, N., Bendall, R., Legrand-Abravanel, F., Xia, N., ljaz, S., Izopet, J., \& Dalton, H. (2012). Hepatitis E. The Lancet, 379(9835), 2477-2488. https://doi.org/10.1016/s0140-6736(11)61849-7

Lhomme, S., Abravanel, F., Dubois, M., Chapuy-Regaud, S., SandresSaune, K., Mansuy, J. M., ... Izopet, J. (2015). Temporal evolution of the distribution of hepatitis $\mathrm{E}$ virus genotypes in Southwestern France. Infection Genetics and Evolution, 35, 50-55. https://doi. org/10.1016/j.meegid.2015.07.028

Lucarelli, C., Spada, E., Taliani, G., Chionne, P., Madonna, E., Marcantonio, C., ... Ciccaglione, A. R. (2016). High prevalence of anti-hepatitis E virus antibodies among blood donors in central Italy, February to March 2014. Eurosurveillance, 21(30). https://doi.org/10.2807/15607917.es.2016.21.30.30299

Purdy, M. A., Harrison, T. J., Jameel, S., Meng, X. J., Okamoto, H., \& der, Van Poel, W. H. M., ... ICTV Report Consortium. (2017). ICTV Virus Taxonomy Profile: Hepeviridae. Journal of General Virology, 98, 26452646. https://doi.org/10.1099/jgv.0.000940

Smith, D., Bradley-Stewart, A., Ijaz, S., Harvala, H., Hogema, B., Izopet, J., ... Simmonds, P. (2015). Variability and pathogenicity of hepatitis $E$ virus genotype 3 variants. Journal of General Virology, 96(11), $3255-$ 3264. https://doi.org/10.1099/jgv.0.000264

Spada, E., Pupella, S., Pisani, G., Bruni, R., Chionne, P., Madonna, E., ... Liumbruno, G. M. (2018). A nationwide retrospective study on prevalence of hepatitis $\mathrm{E}$ virus infection in Italian blood donors. Blood Transfusion, 16(5), 413-421. https://doi.org/10.2450/2018.0033-18

Wang, Y., Zhang, H., Ling, R., Li, H., \& Harrison, T. J. (2000). The complete sequence of hepatitis $\mathrm{E}$ virus genotype 4 reveals an alternative strategy for translation of open reading frames 2 and 3. Journal of General Virology, 81, 1675-1686. https://doi. org/10.1099/0022-1317-81-7-1675

Wang, Y. C., Zhang, H. Y., Xia, N. S., Peng, G., Lan, H. Y., Zhuang, H., ... Harrison, T. J. (2002). Prevalence, isolation, and partial sequence analysis of hepatitis $E$ virus from domestic animals in China. Journal of Medical Virology, 67, 516-521. https://doi.org/10.1002/jmv.10131

WHO. Hepatitis E. (2018). Retrieved from http://www.who.int/ mediacentre/factsheets/fs280/en/

Wu, J., Si, F., Jiang, C., Li, T., \& Jin, M. (2015). Molecular detection of hepatitis $E$ virus in sheep from southern Xinjiang, China. Virus Genes, 50, 410-417. https://doi.org/10.1007/s11262-015-1194-9

Yan, B., Zhang, L., Gong, L., Lv, J., Feng, Y., Liu, J., ... Xu, A. (2016). Hepatitis E virus in yellow cattle, Shandong, Eastern China. Emerging Infectious Diseases, 22(12), 2211-2212. https://doi.org/10.3201/ eid2212.160641

How to cite this article: Sarchese V, Di Profio F, Melegari I, et al. Hepatitis E virus in sheep in Italy. Transbound Emerg Dis. 2019;00:1-6. https://doi.org/10.1111/tbed.13157 